\title{
Molecular dynamics simulation of microcrack healing in copper
}

\author{
S. Li ${ }^{\text {a }}$, K.W. Gao ${ }^{\text {a }}$, L.J. Qiao ${ }^{\text {a,* }}$, F.X. Zhou ${ }^{\text {b }}$, W.Y. Chu ${ }^{\text {a }}$ \\ ${ }^{a}$ Department of Materials Physics, University of Science and Technology, Beijing 100083, People's Republic of China \\ ${ }^{\mathrm{b}}$ LNM Institute of Mechanics, CAS, Beijing 100081, People's Republic of China
}

Received 27 April 2000; received in revised form 18 July 2000; accepted 21 August 2000

\begin{abstract}
The molecular dynamics method is used to simulate microcrack healing during heating or/and under compressive stress. A center microcrack in $\mathrm{Cu}$ crystal could be sealed under a compressive stress or by heating. The role of compressive stress and heating in crack healing was additive. During microcrack healing, dislocation generation and motion occurred. If there were pre-existed dislocations around the microcrack, the critical temperature or compressive stress necessary for microcrack healing would decrease, and the higher the number of dislocations, the lower the critical temperature or compressive stress. The critical temperature necessary for microcrack healing depended upon the orientation of crack plane. For example, the critical temperature of the crack along (001) plane was the lowest, i.e., 770 K. (c) 2001 Elsevier Science B.V. All rights reserved.
\end{abstract}

Keywords: Microcrack healing; Molecular dynamics simulation; Dislocation; $\mathrm{Cu}$

\section{Introduction}

The microcracks in ceramic [1-3], plexiglass [4] and ice [5] could be healed through atom diffusion during heating. Microcrack in metal materials can also be healed. For example for hot-rolled Inconel 600 alloy, microcracks initiating early at the grain boundary were closed during heating over $870^{\circ} \mathrm{C}$ with a large compressive stress by moving grain boundaries because of recrystallization [6]. For $\mathrm{Cu} 30 \mathrm{Fe}$ duplex alloy, microcracks forming early during rolling at room temperature could be closed after rolling exceeded 30\% [7]. For 20 MnMo steel, microcracks disappeared during hot deformation

\footnotetext{
${ }^{*}$ Corresponding author.

E-mail address: lqiao@ustb.edu.cn (L.J. Qiao).
}

[8]. In situ TEM observations showed that for metal materials, whether ductile or brittle, dislocation emission and motion occurred before and during crack initiation or/and propagation [9-11]. Therefore, the resistance for crack propagation is $2 \gamma+\gamma_{p}$, where $\gamma$ is the surface energy and $\gamma_{p}$ the plastic work. What remains to be elucidated is whether dislocation emission and motion are involve in microcrack closure or healing; or plastic deformation is also involved in the resistance required for microcrack healing?

Dislocation emission and crack propagation can be simulated by molecular dynamics method [12-14]. In the present paper, the molecular dynamics method is used to simulate microcrack healing during pressing or heating, and to exhibit the role of the plastic deformation in microcrack healing. 


\section{Computation procedure}

Copper was chosen as the simulated material. The inter-atomic potential used here is the N-body potential proposed by Finnis and Sinclair [15] according to the embedded atom method (EAM) and constructed by Ackland et al. [16]. The inner atoms follow law of Newton, and the leapfrog algorithm [17] is applied to calculate positions and velocities of atoms. The initial velocity is the Maxwell-Boltzmann distribution corresponding to a given temperature. The system temperature is maintained at a constant value by scaling the atom velocities during the simulations.

The $x$ axis is along the extension line of the center crack, the $y$ along the normal of the crack

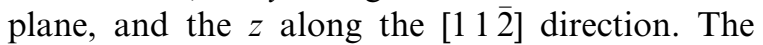
inclination angle $\theta$ between the (1111) slip plane and the crack plane changes from $0^{\circ}$ to $90^{\circ}$, and in general, is set to be $70^{\circ}$, as shown in Fig. 1(a), where $a_{0}$ is the lattice constant, the length of the crystal along the $x$ axis direction is $45 \times \sqrt{2} / 2 a_{0}$. The width along the $y$ axis direction is $25 \times \sqrt{3} a_{0}$, and the thickness along the $z$ axis direction is $\sqrt{2} / 2 a_{0}$. The number of atoms used here is about 6000 . The length of the center crack is $2 a=3.6$ $\mathrm{nm}$, and width is $0.6 \mathrm{~nm}$, which is larger than the potential cutoff distance, which is $0.44 \mathrm{~nm}$.

During the heating, a fixed boundary condition is used in the $x$ and $y$ directions. That is, the locations of the boundary atoms are remained unchanged during the whole calculation process. During loading and heating under a compressive stress, the displacement boundary condition is used in the $x$ and $y$ directions. That is, the boundary atoms move according to the plane strain linear elastic displacement field for a cracked solid [18]. Along the $z$ direction, a six layer periodic representation is applied in all simulation.

The edge-cracked crystal is easy to emit dislocation during mode I loading [12-14] but cannot be closed. For the center-cracked crystal, the situation is quite opposite. In order to generate dislocations in the center-cracked crystal, the edgecracked crystal is to be used to emit dislocations under mode I loading, and then the atomic configuration in the area containing the dislocations, which contains $24 \times 24$ rows atoms, is copied to

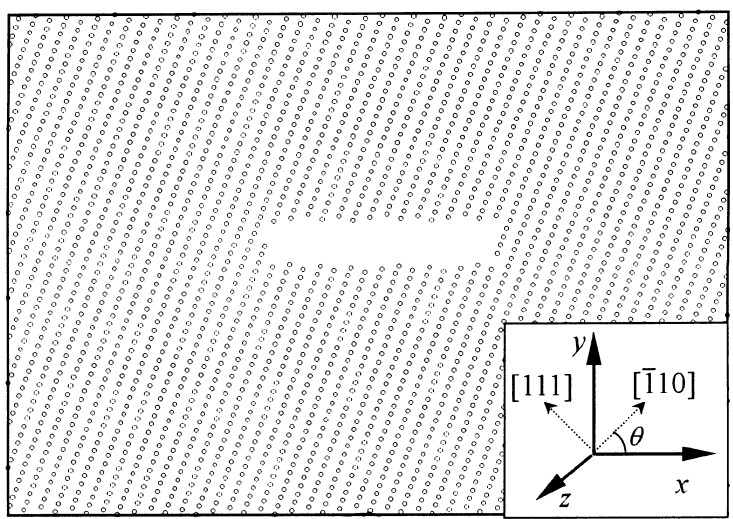

(a)

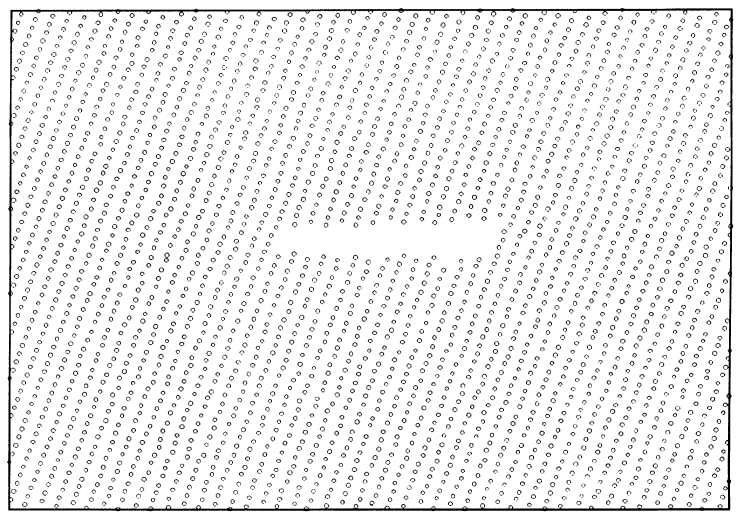

(b)

Fig. 1. The atomic configuration obtained by relaxing at $40 \mathrm{~K}$ (a) and at $700 \mathrm{~K}$ (b).

the center-cracked crystal. During coping, the relative displacements between boundary atoms of the area containing the dislocations and their nearest atoms in the edge-cracked crystal are the same as those in the center-cracked crystal.

\section{Results and discussion}

\subsection{Crack healing by heating}

The inclination angle $\theta$ between the crack plane and the (111) slip plane is $70^{\circ}$. After the center crack is formed, the configuration is relaxed at $40 \mathrm{~K}$, which is near to $0 \mathrm{~K}$, until reaching 
equilibrium, as shown in Fig. 1(a). The temperature is chosen to be $600,650,700,750$ and $800 \mathrm{~K}$, respectively, and maintained at a constant during relaxation. The time step is $7.5 \times 10^{-4}$ ps. Simulations show that when temperature is maintained at 600,650 and $700 \mathrm{~K}$, the center crack cannot close after relaxing for $50 \mathrm{ps}$, which is a relatively long time, as shown in Fig. 1(b). If the temperature is maintained at $750 \mathrm{~K}$, the crack begins to heal after relaxing for 3 ps along with generating dislocations, as shown in Fig. 2(a). After 7.5 ps, the crack is approximately healed, but a void $V$ and some dislocations as $B, C$, and $D$ are left, as shown in Fig. 2(b). The void does not disappear after relaxing for $50 \mathrm{ps}$ at $750 \mathrm{~K}$. At $800 \mathrm{~K}$, the crack is closed completely after relaxing for $9 \mathrm{ps}$, and some dislocations as $A, B, C, D, E, F$ and $G$ but no void are left, as shown in Fig. 2(c).

\subsection{Crack healing by compressive stress}

Remaining the temperature at $40 \mathrm{~K}$, a compressive stress $\sigma$ perpendicular to the crack plane is applied with a loading rate of $0.13 \mathrm{GPa} / \mathrm{ps}$. When the compressive stress increases to $\sigma=7.26 \mathrm{GPa}$, the crack heals to some extent, but a void and some dislocations are left, as shown in Fig. 3(a). When the compressive stress is increased to $\sigma=13.2 \mathrm{GPa}$, the crack closes completely, and some dislocations but no void remain in the crystal, as shown in Fig. 3(b).

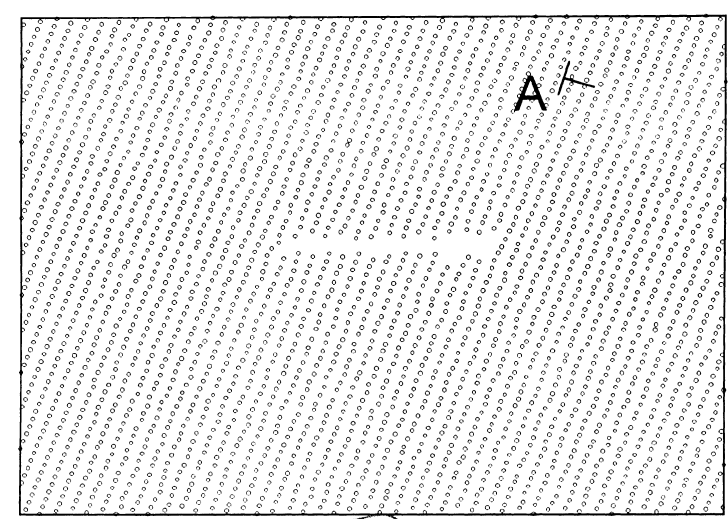

(a)

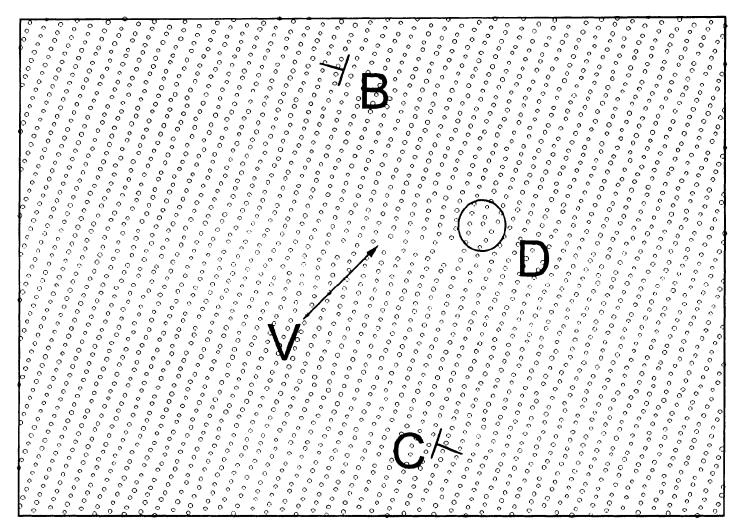

(b)

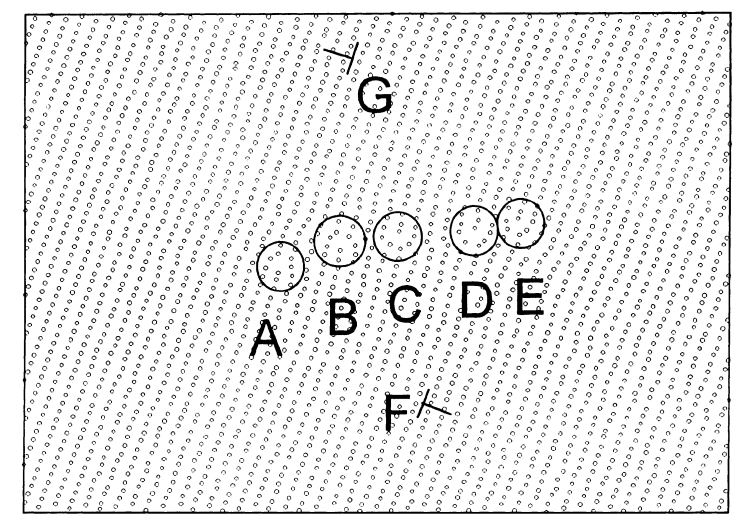

(c)

Fig. 2. Crack healing process. After relaxing for 3 ps (a), $7.5 \mathrm{ps}$ (b) at $750 \mathrm{~K}$, and for 9 ps at $800 \mathrm{~K}$ (c). $A$ to $G$ are dislocations and $V$ void. 


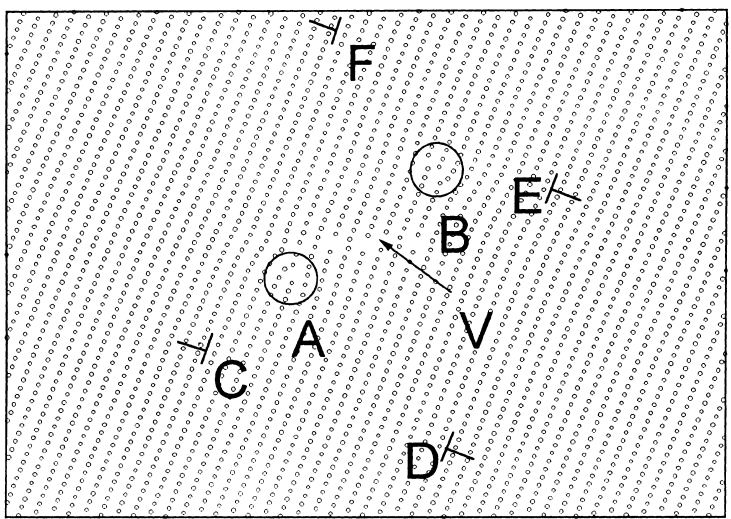

(a)

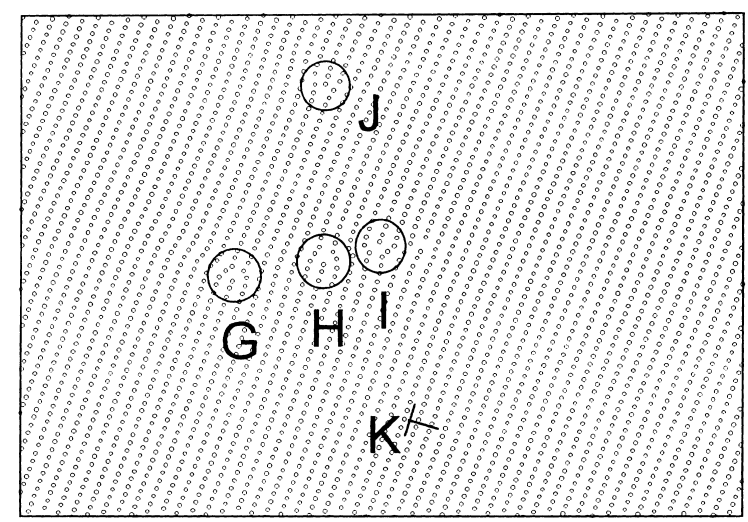

(b)

Fig. 3. Crack healing under compressive stress of $7.26 \mathrm{GPa}$ (a) and $13.2 \mathrm{GPa}$ (b) at $0 \mathrm{~K}$. $A$ to $K$ are dislocations, $V$ void.

\subsection{Crack healing during heating under compressive stress}

Simulations show that the critical compressive stress and critical temperature necessary for crack healing are $\sigma_{\mathrm{c}}=13.2 \mathrm{GPa}$ and $T_{\mathrm{c}}=800 \mathrm{~K}$, respectively. If compressive stress $\sigma=3.96 \mathrm{GPa}$ is applied and temperature is maintained at $600 \mathrm{~K}$, the crack is closed completely after relaxing for $11.25 \mathrm{ps}$ and some dislocations are left, as shown in Fig. 4. Therefore, the applied compressive stress of $3.96 \mathrm{GPa}$ can decrease the critical temperature necessary for crack healing from 800 to $600 \mathrm{~K}$. Fig. 4 also indicates that increasing temperature from 40 to $600 \mathrm{~K}$ can reduce the critical compressive stress necessary for crack healing from

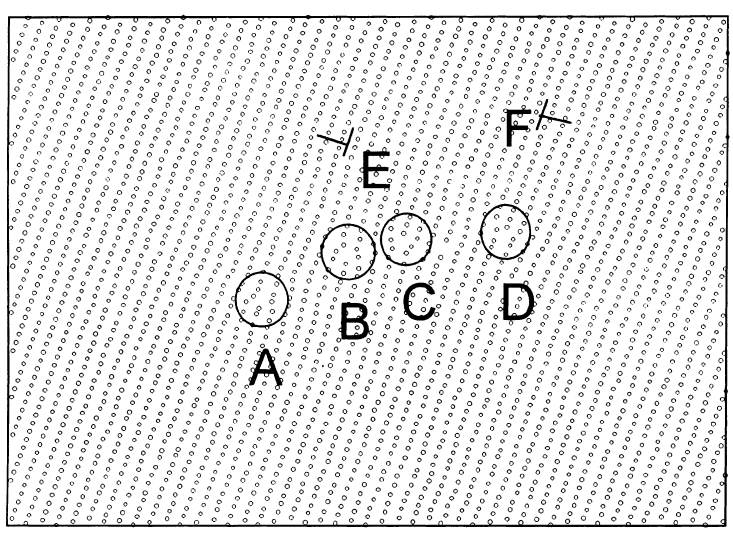

Fig. 4. Crack healing at $600 \mathrm{~K}$ under $\sigma=3.96 \mathrm{GPa}, A$ to $D$ are Frank dislocations, $E$ and $F$ Shockley dislocations.

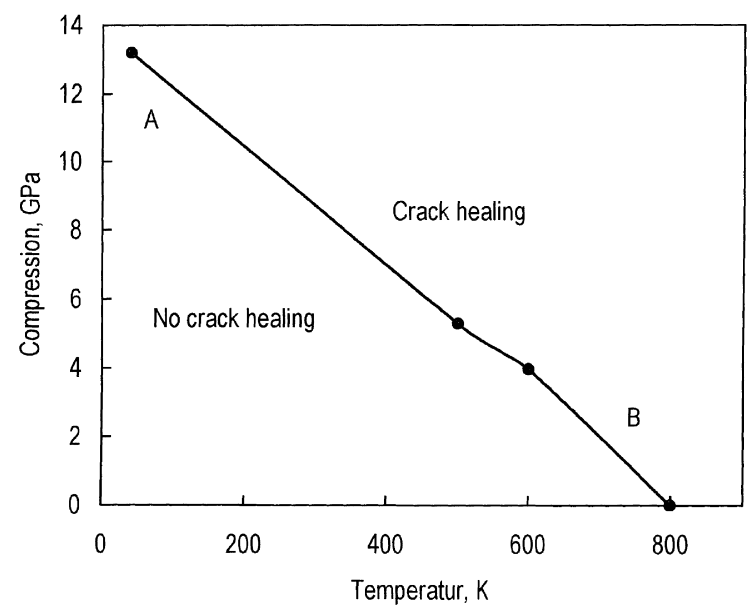

Fig. 5. The regions of crack healing and no crack healing in the coordinate system of temperature and compressive stress.

13.2 to $3.96 \mathrm{GPa}$. If the compressive stress is 5.28 $\mathrm{GPa}$, the temperature for crack healing is $500 \mathrm{~K}$. These results indicate that the role of compressive stress and heating in crack healing is additive. Fig. 5 shows that the crack healing can occur above the curve $A B$, and no crack healing under the curve $A B$.

\subsection{Effect of orientation of crack plane on crack healing}

If the crack plane is along the (1 111$)$ slip plane $(\theta=0)$, the critical temperature necessary for 
crack healing is $850 \mathrm{~K}$ instead of $800 \mathrm{~K}$, as shown in Fig. 6(a). Crack healing at $850 \mathrm{~K}$ is also accompanied with the generation and movement of dislocations.

If the crack plane is along ( 0001$)$ plane $\left(\theta=54.7^{\circ}\right)$, the critical temperature of crack healing is $770 \mathrm{~K}$, as shown in Fig. 6(b). When the crack plane is along $(\overline{1} 10)$ plane $\left(\theta=90^{\circ}\right)$, the critical temperature of crack healing is $790 \mathrm{~K}$, as shown in Fig. 6(c).

\subsection{Effect of dislocation on crack healing}

For the edge-cracked crystal, a tensile stress perpendicular to the crack plane is applied with a loading rate of $0.079 \mathrm{GPa} / \mathrm{ps}$. When $\sigma_{\mathrm{I}}=3.95 \mathrm{GPa}$ corresponding to 10000 time steps, the first Shockley dislocation is emitted from the crack tip, and when $\sigma_{\mathrm{I}}=4.74 \mathrm{GPa}$ corresponding to $K_{\mathrm{I}}=0.6 \mathrm{MPam}^{1 / 2}$, two Shockley dislocations are emitted from the tip of the edge crack, as shown in Fig. 7(a). The atomic configuration of the edgecracked crystal in area $A$, which contains the two dislocations, is copied to the center-cracked crystal, as shown in Fig. 7(b).

For the center-cracked crystal containing no dislocation, the critical temperature for crack healing is $800 \mathrm{~K}$, as shown in Fig. 2(c). However, when the center-cracked crystal containing two Shockley dislocations is maintained at $650 \mathrm{~K}$, the crack begins to heal after 2500 time steps, at the same time, the pre-existing dislocations have disappeared and some new dislocations have

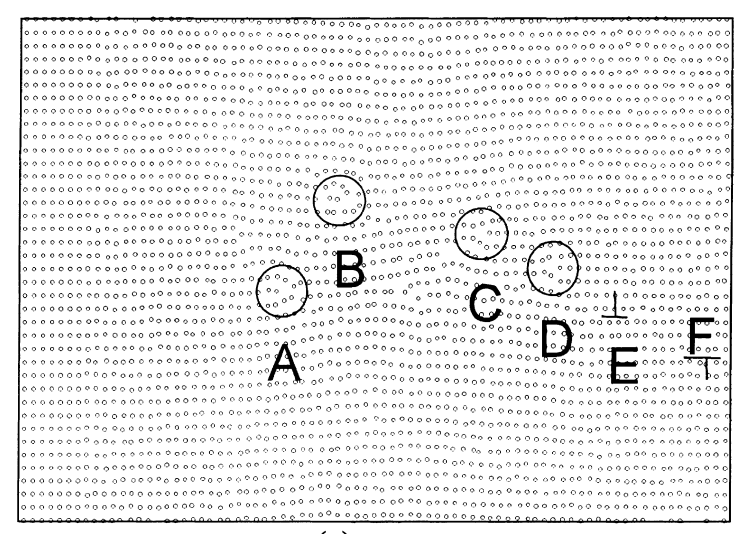

(a)

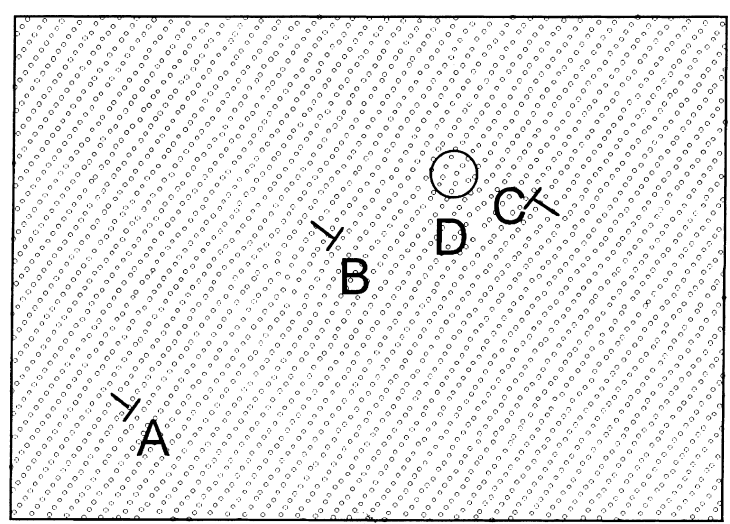

(b)

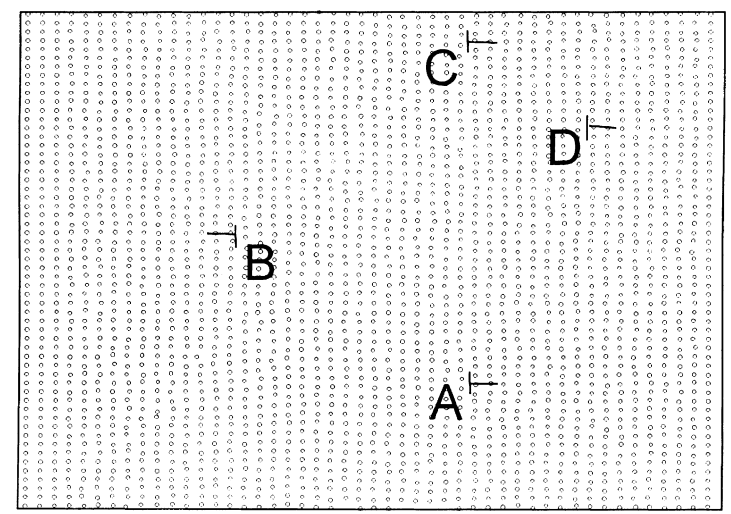

(c)

Fig. 6. Healing of the crack along (1 111$)$ plane at $850 \mathrm{~K}$ (a), ( $\left.\begin{array}{lll}0 & 1\end{array}\right)$ plane at $770 \mathrm{~K}$ (b) and (1 10$)$ plane at $790 \mathrm{~K}$ (c). 


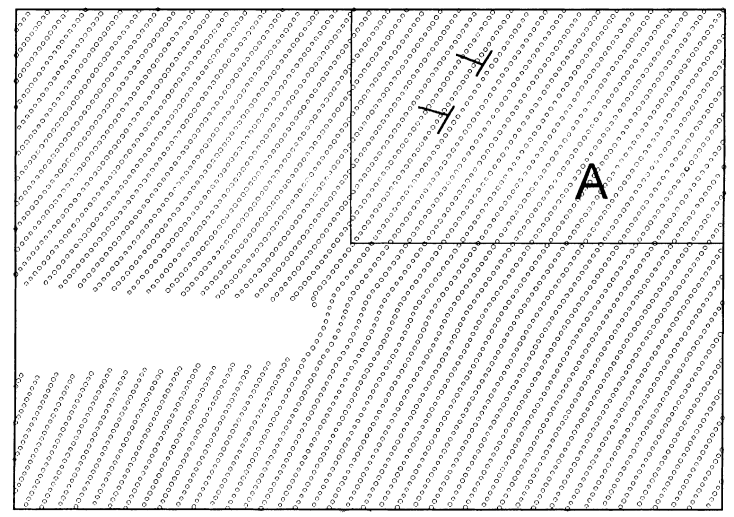

(a)

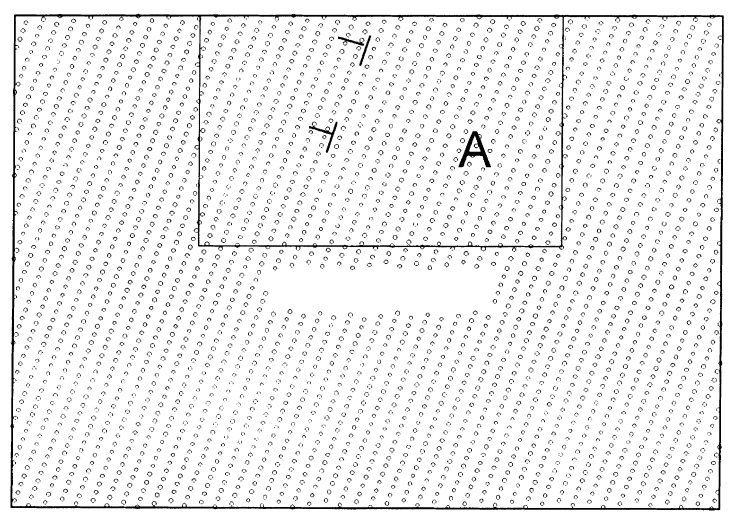

(b)

Fig. 7. The atomic configuration containing two dislocations, (a) edge crack, (b) center crack.

appeared. After relaxing at $650 \mathrm{~K}$ for 10000 time steps, the crack is closed completely and some new dislocations form, as shown in Fig. 8(a). This shows that two pre-existing dislocations can reduce the critical temperature necessary for crack healing from 800 to $650 \mathrm{~K}$. For the centercracked crystal without dislocations, the critical compressive stress necessary for crack healing at $40 \mathrm{~K}$ is $\sigma=13.2 \mathrm{GPa}$, as shown in Fig. 3(b). If there are two pre-existing dislocations, the crack will be closed completely under a compressive stress $\sigma=9.9 \mathrm{GPa}$ at $40 \mathrm{~K}$, as shown in Fig. 8(b). This indicates that two pre-existing dislocations can decrease the critical compressive stress for crack healing from 13.2 to $9.9 \mathrm{GPa}$. In a word, pre-existed dislocations can promote crack healing. If the atomic configuration in the area $A$ shown in Fig. 7(a) is copied twice to the center-cracked crystal, there are four pre-existing dislocations. At $500 \mathrm{~K}$, the crack has been completely closed after relaxing for 6500 time steps and some dislocations are generated, as shown in Fig. 8(c). Therefore, pre-existing dislocation can decrease the critical temperature for crack healing and the higher the number of the pre-existing dislocations, the lower the critical temperature for crack healing.

Applied compressive stress $\sigma$ can cause crack healing, as shown in Fig. 3, therefore, the elastic energy $\sigma^{2} \pi a\left(1-v^{2}\right) / E$ is a driving force for crack healing. On the other hand, heating can cause crack healing, hence thermal energy is also the driving force for crack healing. Fig. 4 indicates that the compressive stress can decrease the critical temperature for crack healing, and increasing the temperature can reduce the critical compressive stress for crack healing. This shows that the two driving forces are additive, as shown in Fig. 5. Fig. 8 shows that pre-existing dislocations could reduce the critical temperature or the critical compressive stress for crack healing. May be the pre-existing dislocations can reduce the resistance of crack healing.

\section{Conclusions}

1. Compressive stress or heating can cause crack healing up and their role is additive.

2. Crack healing is accompanied with the generation and movement of dislocations.

3. The critical temperature of crack healing is related with the orientation of crack plane.

4. Pre-existing dislocations can reduce the critical temperature necessary for crack healing.

\section{Acknowledgements}

The present work was supported by the Special Funds for the Major State Basic Research Projects (No. G19990650) and by the National Natural Science Foundation of China (No. 19891180, 59871010). 


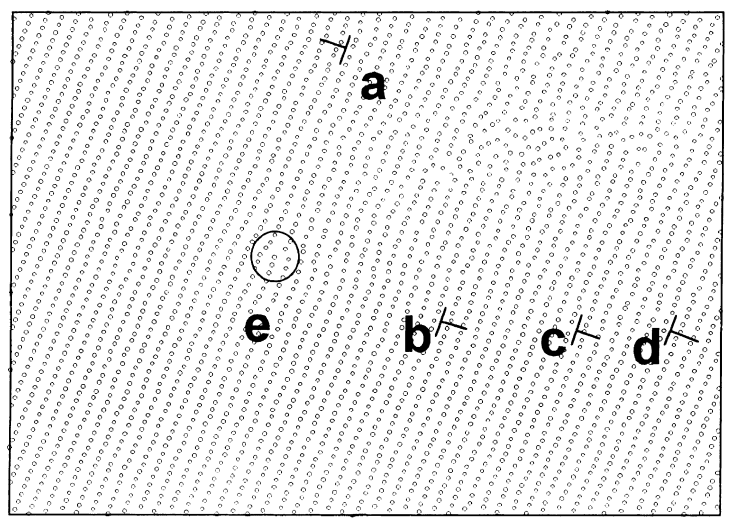

(a)

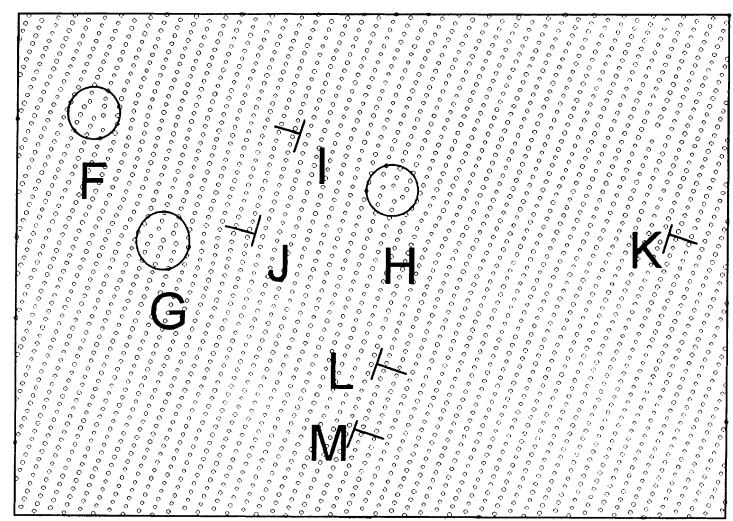

(b)

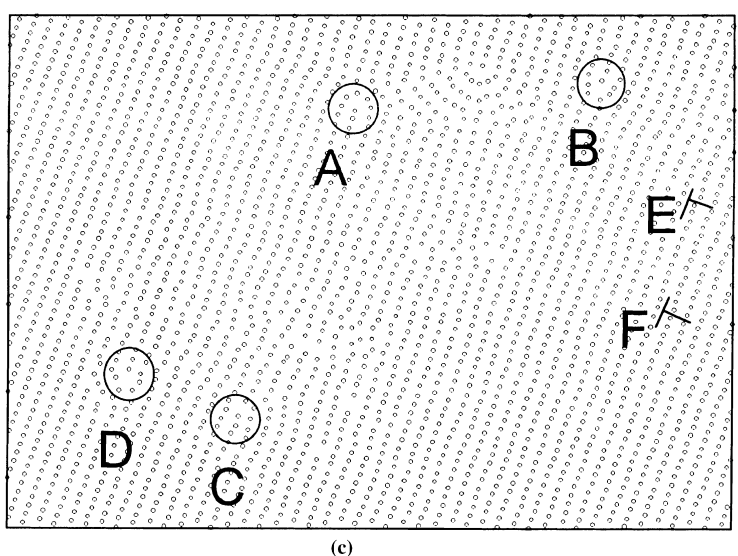

Fig. 8. Crack healing for the crystal containing two dislocations at $650 \mathrm{~K}$ (a), at $0 \mathrm{~K}$ but under a compressive stress of $9.9 \mathrm{GPa}$ (b), and containing four dislocations at $500 \mathrm{~K}$ (c).

\section{References}

[1] Z. Wang, Y.Z. Li, M.P. Harmer, Y.T. Chou, J. Amer. Ceram. Soc. 74 (1992) 1596.

[2] T.K. Gupta, J. Amer. Ceram. Soc. 59 (1976) 259.

[3] A.M. Thompson, H.M. Chan, M.P. Harmer, R.F. Cook, J. Amer. Ceram. Soc. 78 (1995) 567.

[4] M.K.C. Holden, V.D. Frechette, Amer. J. Ceram. Soc. 72 (1989) 2189.

[5] S.C. Colbeck, Acta Metall. 34 (1) (1986) 89.

[6] E. Shapiro, G.E. Dietar, High temperature high strain rate fracture of Inconed 600, Metall. Trans. 1 (1970) 1711.

[7] J. Foct, N. Akdut, G. Gottstein, Scripta Meta. Mater. 27 (1992) 1033.

[8] J.T. Han, G. Zhao, Q.X. Cao, A regularity of crack healing for 20MnMo steel, Sci., China 27E (1997) 23.

[9] Q.Z. Chen, W.Y. Chu, C.M. Hsiao, In situ TEM Observations of nucleation and bluntness of nanocracks in thin crystal of 310 stainless steel, Acta Metall. 43 (1995) 4371.
[10] Q.Z. Chen, K.W. Gao, Y. Zhang, W.Y. Chu, Nucleation blunting and propagation of a nanocrack in DFZ of thin crystals, Fat. Fract Eng. Mater. Struct. 21 (1998) 1415 .

[11] K.W. Gao, W.Y. Chu, Y.B. Wang, In situ TEM observation of dislocation-enhanced dislocation emission, motion and the nucleation of SCC for Ti-24Al-11 Nb alloy in methanol, Scripta Mater. 36 (1997) 259.

[12] R.G. Hoagland, M.S. Daw, S.M. Foiles, M.I. Baskes, An atomic model of crack tip deformation in $\mathrm{Al}$ using an embedded atom potential, J. Mater. Sci. 5 (1990) 313.

[13] Y.W. Zhang, T.C. Wang, Q.H. Tang, Brittle and ductile fracture at the atomistic crack tip in copper crystals, Scripta Mater. 33 (1995) 267.

[14] H. Kitagawa, A. Nakatani, Y. Shibutani, Molecular dynamics study of crack processes associated with dislocation nucleated at the tip, Mater. Sci. Eng. A 176 (1994) 263. 
[15] M.W. Finnis, J.E. Sinclair, A simple N-body potential for transition metals, Philos. Mag. A 50 (1984) 45.

[16] G.J. Ackland, G. Tichy, V. Vitek, M.W. Finnis, Simple Nbody potentials for the noble metals and nickle, Philos. Mag. A 56 (1987) 735.
[17] D.W. Heermann, Computer Simulation Methods in Theoretic Physics, 2nd ed., Springer, Berlin, 1990.

[18] J.F. Knott, Fundamentals of Fracture Mechanics, Butterworth, London, 1973, p. 54. 